\title{
Longitudinal Jerk Estimation of Driver Intentions for Advanced Driver Assistance Systems
}

\author{
Andrea Bisoffi, Francesco Biral, Mauro Da Lio, and Luca Zaccarian
}

\begin{abstract}
This work aims at estimating the longitudinal jerk of the vehicle as it is acted by a human driver, in the context of preventive safety. A reliable estimate is needed to infer the current driver intention in an advanced driving assistance system developed by the authors. The derived intention-oriented model for the longitudinal dynamics is embedded into an enhanced Kalman filter that provides the user with a knob to trade off between responsiveness of the estimate and noise rejection. The scheme is fit for on-line usage, relies on signals commonly available on the CAN bus of modern vehicles, and requires a very limited number of parameters. Its effectiveness is validated on experimental data, and compared with alternative approaches.
\end{abstract}

\section{INTRODUCTION}

$\mathbf{J}$

ERK, the time derivative of acceleration, plays an important role in human movement. In particular, it was shown (see, e.g., [1], [2]) that jerk minimization is one of the rationales behind human movement planning (also related to minimum variance control under proportional neural noise [3]). Minimizing the jerk yields the smoothest trajectory that accomplishes the given kinematic goals [1, p. 1689].

From robotics to automotive, jerk minimization is reasonably sought by human drivers when they determine their trajectory (see [4], [5]), possibly weighting also the maneuvering time in their objective [4, Simulations]. In particular, it was shown experimentally in [5, §4.3.2] that voluntary driving maneuvers (such as braking and steering) synchronize with bursts in the jerk signal. [6] exploited the informativeness of the jerk about the strategy behind a driver maneuver to formulate a new vehicle control, starting from the jerk behaviour of an expert driver.

Based on these results, an Advanced Driver Assistance System (ADAS) called co-driver [7] was devised within the European project interactIVe [8] in order to assess the level of risk of the current maneuver pursued by the driver, and to issue suitable warnings. Using the information from the environment, the co-driver hypothesizes a set of different (short-term, high-level) driving maneuvers, each of which maps to a different jerk evolution. Then the co-driver assumes by a mirroring process that the current driver maneuver is the one in the set whose jerk has the least mismatch [7, §III.D.6), Eq. (26)] with the jerk associated to the driver, called driver intentional jerk. In this way, the co-driver infers on line the driver intention (in the sense of [9]) and assists the driver if needed.

Accurate estimates of the driver intentional jerk are hence decisive because they improve the driver intention inference based on the comparison with the co-driver hypotheses, reducing the probability of false or missed alarms.

The previous discussion sets out the main motivation for this work. Our objective is to obtain an on-line estimate of the longitudinal jerk as it is acted by the typical human driver, in the context of preventive safety, away from the maneuvrability limits.

We discuss below the possible approaches to estimate the driver intentional longitudinal jerk. Although a jerk sensor has been proposed (its working principle is described in [10, $\S 4]$ ), a more viable solution is to obtain the jerk by suitably filtering the longitudinal acceleration, like in [11, $\S \mathrm{IV} . \mathrm{B}]$ or $[6, \S 5.2]$, together with the improved methods [12], [13]. Alternatively, one could use high-gain techniques applied to numerical differentiation, as in [14] and [15].

However, the longitudinal acceleration of a vehicle includes not only the effect of pedal actions, to which the intention is strongly related, but also the driveline dynamics, vehicle vibrations, the road slope and so forth, which are to be considered as noise with respect to driver intentions. Therefore, we need to combine the acceleration measurement (preliminarily deprived of the road slope and static biases) with the gas and brake pedal signals, which are the closest expression of the driver intention. Then, a natural solution to achieve a virtual driver-intentional-jerk sensor is Kalman filtering (see, e.g., [16] and [17], where the schemes are tested in simulations).

We further enrich our Kalman formulation by a "high"-gain-inspired scaling, through the scaling parameter $\ell$. Whereas "high"-gain approaches [18] typically take $\ell$ large ("high") to overcome nonlinear effects, here we just exploit their key idea of moving through scaling the location of all the eigenvalues of the error dynamics, which were initially placed with a Kalman approach. In this way, $\ell$ acts as a tuning knob allowing easily for trade-offs between convergence rate of the estimation error and sensitivity to noise. Our approach relies strongly on physical considerations, wich justify a dynamical model for the driver

A. Bisoffi, F. Biral and M. Da Lio are with Dipartimento di Ingegneria Industriale, University of Trento, Italy. L. Zaccarian is with Dipartimento di Ingegneria Industriale, University of Trento, Italy and CNRS, LAAS and Université de Toulouse, 7 Avenue du Colonel Roche, 31077 Toulouse, France. Email: \{andrea.bisoffi, francesco.biral, mauro.dalio\}@unitn.it, zaccarian@laas.fr 
intentional jerk. For this reason, an enhanced Kalman filter seems to be a more promising first approach than other statistical filtering. Investigating these alternative approaches in the present context is left as future work.

The paper is organized as follows. In Section II we present a suitable model for the driver intention based on pedal signals. Section III builds the scheme that estimates the driver intentional jerk, based on a "high"-gain enhancement of a Kalman formulation. Section IV validates our estimation scheme on experimental data from a road test, by comparison with other possible solutions and for different drivers.

A preliminary version of this work was presented in [19], where we investigated the feasibility of this approach only in the case of traction, and the validation was performed on a much smaller data set. On the other hand, here we generalize our filtering scheme to include the case of braking, and use directly pedal signals that are conceptually closer to the driver intention. To use these signals effectively, we add an identification part in Section IV-A. Thanks to the larger amount of data (also from different drivers), a more thorough validation of the generalized scheme is then possible in Sections IV-B, IV-C and IV-D.

\section{LONGITUDINAL VEHICLE DYNAMICS AND DRIVER INTENTION}

In this section we first present a standard model for the longitudinal vehicle dynamics, and then we show how to reformulate it in such a way that the driver intentions are suitably considered. The obtained equations represent the model-based part of the enhanced Kalman formulation of the next section.

\section{A. Model for the longitudinal vehicle dynamics}

From Newton's equation, the longitudinal dynamics of a vehicle reads

$$
M \dot{v}=\tau T_{e}-F_{b}-\underbrace{M g \sin (\alpha)}_{=: F_{s}}-\underbrace{C_{r} M g \cos (\alpha)}_{=: F_{f}}-\underbrace{\frac{1}{2} \rho S C_{x} v^{2}}_{=: F_{a}}
$$

where $v$ is the longitudinal velocity of the vehicle, $F_{b}$ is the brake force, $\tau T_{e}$ is the force actuated by the engine on the wheels via the gearbox and the driveline, through the transmission coefficient

$$
\tau:=\frac{1}{r_{r}} \eta_{g} \eta_{d} \tau_{g} \tau_{d}
$$

In (1a), $F_{s}, F_{f}$ and $F_{a}$ are respectively the dissipative forces due to slope $\alpha$, friction, and aerodynamic drag. All the remaining terms are constant physical parameters that are listed in Table I.

Table I

PHYSICAL PARAMETERS FOR THE LONGITUDINAL DYNAMICS.

\begin{tabular}{ll}
\hline Parameter & Symbol \\
\hline Vehicle mass & $M$ \\
Rolling radius & $r_{r}$ \\
Gearbox efficiency & $\eta_{g}$ \\
Driveline efficiency & $\eta_{d}$ \\
Gearbox transmission ratio & $\tau_{g}$ \\
Driveline transmission ratio & $\tau_{d}$ \\
Gravity & $g$ \\
Rolling friction coefficient & $C_{r}$ \\
Air density & $\rho$ \\
Reference area & $S$ \\
Drag coefficient & $C_{x}$ \\
\hline
\end{tabular}

In urban and extra-urban roads not involving extreme mountain routes, the road slope $\alpha$ can be assumed to be sufficiently small, which allows us to approximate (1a) with

$$
M \dot{v}=\tau T_{e}-F_{b}-M g \alpha-C_{r} M g-\frac{1}{2} \rho S C_{x} v^{2} .
$$

The measured quantities are the acceleration (from an accelerometer) and the longitudinal velocity (from the odometers), which are respectively given by

$$
\begin{aligned}
& y_{1}=\dot{v}+g \sin (\alpha)+\bar{d}_{a} \simeq \dot{v}+g \alpha+\bar{d}_{a} \\
& y_{2}=v .
\end{aligned}
$$

The accelerometer measurement in $(2 b)$ is partially corrupted by the road slope $g \alpha$ and the (possible) static misalignment of the sensor $\bar{d}_{a}$. Measuring the longitudinal velocity through odometers in (2c) neglects possible slip phenomena, but since they lie beyond the actuation capabilities of humans and then they can not be associated to driver intentions, we accept this approximation. 


\section{B. Acceleration model for driver intention}

In the following we are considering a vehicle operating with a robotized gearbox and an electronic clutch. In this setting, the driver acts on the gas pedal position $p_{r}$ and on the cylinder brake pressure $p_{b} . p_{r}$ is converted to a torque request $T_{r}$. Most of the time, the actuated torque $T_{e}$ coincides with $T_{r}$ (neglecting the much faster engine dynamics), but the engine control unit may enforce $T_{e} \neq T_{r}$, based on the whole state of the engine/powertrain (for instance, to smooth out gear shifts).

The objective of this work is to estimate the driver intentional jerk. So, we proceed in two steps: first we derive an intentionoriented acceleration model building on the signals $T_{r}$ and $F_{b}$. Second, we trace back these two signals respectively to $p_{r}$ and $p_{b}$, from which $T_{r}$ and $F_{b}$ originate, and which we can easily access.

Define the dissipative force $F_{d}$ as the following sum

$$
F_{d}(v):=F_{s}+F_{f}+F_{a}(v)=M g \alpha+C_{r} M g+\frac{1}{2} \rho S C_{x} v^{2}
$$

and the logical variable $\sigma$ as

$$
\sigma(t):= \begin{cases}1 & \text { if the driver is braking at time } t \\ 0 & \text { otherwise. }\end{cases}
$$

This leads to two intention-oriented acceleration sub-models. When the driver is not braking $(\sigma=0)$, that is, s/he is accelerating or not pressing any pedal, the only relevant intention arises from the torque request $T_{r}$. If $T_{r}$ were actuated directly by the engine with no in-between interposition, we would obtain an intentional acceleration $\dot{v}_{i, 0}$ as

$$
M \dot{v}_{i, 0}=\tau T_{r}-F_{d}(v) \quad(\sigma=0) .
$$

We write $F_{d}(v)$ (and not $F_{d}\left(v_{i, 0}\right)$ ) because the driver is able to compensate approximately for these macroscopic dissipative effects through part of its action $T_{r}$ (this compensation through his/her control action is linked to feedback linearization [20, $\S 2.4 .3])$.

Conversely, when the driver is braking $(\sigma=1)$, the only relevant intention arises from the braking force $F_{b}$ and for a standard driver this intention is mutually exclusive with the torque request $T_{r}$. The direct application of only $F_{b}$ then leads to the intentional acceleration $\dot{v}_{i, 1}$ as

$$
M \dot{v}_{i, 1}=-F_{b}-F_{d}(v) \quad(\sigma=1) .
$$

For all times, then, the intentional acceleration $a_{i}$ is

$$
a_{i}:=(1-\sigma) \dot{v}_{i, 0}+\sigma \dot{v}_{i, 1} .
$$

In (6) we first substitute (5a)-(5b) and then (2a) to eliminate $F_{d}$ defined in (3), as follows:

$$
\begin{aligned}
M a_{i} & =\tau T_{r}(1-\sigma)-\sigma F_{b}-F_{d}(v) \\
& =\tau T_{r}(1-\sigma)+F_{b}(1-\sigma)-\tau T_{e}+M \dot{v} .
\end{aligned}
$$

As a second step to obtain an intention-oriented acceleration model, we introduce now in (7) the gas pedal position $p_{r}$ and the cylinder brake pressure $p_{b}$ that are directly generated by the driver. $p_{b}$ represents well the driver intentions in the context of preventive safety and for a typical human driver because away from maneuvrability limits the anti-lock braking system does not intervene and the driver has full authority on the brake. We assume the linear dependencies

$$
\begin{aligned}
& F_{b}:=c_{b} p_{b} \\
& T_{r}:=c_{r} p_{r},
\end{aligned}
$$

which write (7) as

$$
\dot{v}=a_{i}-\frac{\tau}{M} c_{r} p_{r}(1-\sigma)-\frac{1}{M} c_{b} p_{b}(1-\sigma)+\frac{\tau}{M} T_{e} .
$$

Section IV-A is devoted to the identification of the two coefficients $c_{b}$ and $c_{r}$, and the results in terms of fitting show the admissibility of the linear dependencies (8a).

\section{Complete model of the driver intention}

We aim at building a state equation that can be used for the model-based part of a Kalman-like filter.

Define

$$
u:=-\frac{\tau}{M} c_{r} p_{r}(1-\sigma)-\frac{1}{M} c_{b} p_{b}(1-\sigma)+\frac{\tau}{M} T_{e},
$$

where $p_{r}, p_{b}, T_{e}$ are measurable on the CAN bus of the vehicle, which is a common feature in modern vehicles, and where $\tau$ is a known function of time because it depends on constant parameters and on the current gear.

With (10a), (9) becomes

$$
\dot{v}=a_{i}+u \text {. }
$$


As jerk is the time derivative of acceleration, the driver intentional acceleration $a_{i}$ and the intentional jerk $\bar{\jmath}_{i}$ satisfy

$$
\dot{a}_{i}=\bar{\jmath}_{i} .
$$

Note that (10c) does not include the contribution of the lateral acceleration in the longitudinal jerk (that is, $\Omega a_{y}$ where $\Omega$ is the yaw rate and $a_{y}$ is the lateral acceleration in the vehicle fixed frame). This is deliberately done since we aim at estimating the driver intentional jerk. From this viewpoint the driver controls the longitudinal force and its rate of change via the throttle and brake pedals. However, the longitudinal force is fixed with the vehicle frame (that is, when the vehicle rotates also the force rotates with it) so we can say that the driver acts on the time derivative of the acceleration in the fixed vehicle frame and consequently only on the second time derivative of the velocity.

The accelerometer offset $o_{a}$ is defined as

$$
o_{a}:=g \alpha+\bar{d}_{a},
$$

that is, it comprises the static misalignment of the accelerometer and the slope of the road, both corrupting the longitudinal acceleration measurement in (2b). Regarding the dynamics of $o_{a}, \dot{\bar{d}}_{a}=0$ is true except from very slow drifts (for example, due to temperature). Since the slope is slowly time varying compared to the other mechanical quantities $v, a$ etc., we also write $\dot{\alpha}=0$. Therefore,

$$
\dot{o}_{a}=0
$$

Finally, we present the equation for the driver intentional jerk. Many studies show that human sensorimotor control is highly optimized, and driven with good approximation by the minimization of the squared jerk [1]-[3], [21]. This principle holds not only for body movements, but also for objects controlled by humans, and cars in particular [4], [5]. Minimization of the squared jerk comes together with minimization of its derivative, because intentional actions are band-limited. We can then assume that human movements aim at minimizing also the squared jerk derivative, by writing

$$
\dot{\bar{j}}_{i}=0
$$

and incorporating the unknown value of $\dot{\bar{j}}_{i}$ into model noise. We set a high value for the variance of the corresponding noise in the Kalman formulation of Section III-A (the fourth term of matrix $Q$ in (21)). Indeed, each state equation in Kalman filters is complemented with a noise term whose variance characterizes the (un)reliability of the corresponding equation (e.g., a higher variance for $(10 \mathrm{e})$ than for (10c) reflects that $(10 \mathrm{e})$ is less reliable than $(10 \mathrm{c}))^{1}$. The approach in (10e) is conceptually the same as the one used when using Kalman filtering solutions for the estimation of the road slope [22]-[25], based on which we wrote $(10 \mathrm{~d})$.

The choice of state equations (10b)-(10e) calls naturally for the state vector $x_{i}:=\left[\begin{array}{lll}v & a_{i} & o_{a} \\ \bar{\jmath}_{i}\end{array}\right]^{T}$, which in turn reformulates (2b) and (2c) as

$$
\begin{aligned}
& y_{1}=a_{i}+o_{a}+u \\
& y_{2}=v,
\end{aligned}
$$

thanks to the definition of $o_{a}$. The consideration after (10e) about equation reliability apply also here. We associate a higher variance to (10f) than to $(10 \mathrm{~g})$ as the former neglects, for instance, pitch motions.

Finally, equations (10) can be represented through the linear dynamical system

$$
\begin{aligned}
\dot{x}_{i} & =A x_{i}+B u \\
y & =C x_{i}+D u,
\end{aligned}
$$

where subscript $i$ stands for intention and

$$
\left[\begin{array}{l|l}
A & B \\
\hline C & D
\end{array}\right]:=\left[\begin{array}{llll|l}
0 & 1 & 0 & 0 & 1 \\
0 & 0 & 0 & 1 & 0 \\
0 & 0 & 0 & 0 & 0 \\
0 & 0 & 0 & 0 & 0 \\
\hline 0 & 1 & 1 & 0 & 0 \\
1 & 0 & 0 & 0 & 1 \\
1 & 0 & 0 & 0
\end{array}\right] .
$$

\section{STATE ESTimation in AN ENHANCED KALMAN APPROACH}

We proceed in two steps. First, we tune a standard Kalman filter for model (11) in Section III-A; second, we build upon this filter and provide in Section III-B a tuning knob to allow the user to choose between responsiveness in the estimation and noise rejection.

The design of the observer is sound by virtue of the observability [20, Chap. 15] of the pair $(C, A)$ in (11c). We note that if we had chosen $\alpha$ and $\bar{d}_{a}$ as separate state variables (instead of the sum $o_{a}=g \alpha+\bar{d}_{a}$ ) together with the state equations $\dot{\alpha}=0$ and $\dot{\bar{d}}_{a}=0$, we would have lost the observability property for the (alternative) corresponding matrices because the contributions of $\alpha$ and $\bar{d}_{a}$ can not be singled out from the output (2b). Nonetheless, distinguishing between $\alpha$ and $\bar{d}_{a}$ is not necessary for the current application.

\footnotetext{
${ }^{1}$ In this quite standard way (see [20, p. 225]), we use the noise variances of the Kalman filter as design parameters.
} 


\section{A. Kalman formulation}

According to the standard Kalman formulation, the state and output equations in (11) are corrupted, respectively, by the noise terms $\Delta_{x}$ and $\Delta_{y}$, as

$$
\begin{aligned}
\dot{x}_{i} & =A x_{i}+B u+\Delta_{x} \\
y & =C x_{i}+D u+\Delta_{y},
\end{aligned}
$$

where the covariance matrices of $\Delta_{x}$ and $\Delta_{y}$ are, through the expected value $E[\cdot]$,

$$
Q:=E\left[\Delta_{x} \Delta_{x}^{T}\right], \quad R:=E\left[\Delta_{y} \Delta_{y}^{T}\right] .
$$

The observer structure for (12) is then

$$
\dot{\hat{x}}_{i}=A \hat{x}_{i}+B u+L\left[y-C \hat{x}_{i}-D u\right]
$$

where $\hat{x}_{i}$ is the estimate of state $x_{i}$. The difference between the last two states defines then the estimation error $\tilde{x}_{i}:=x_{i}-\hat{x}_{i}$, whose dynamics reads, in the absence of the external perturbations $\Delta_{x}$ and $\Delta_{y}$,

$$
\dot{\tilde{x}}_{i}=(A-L C) \tilde{x}_{i} .
$$

Given the plant matrices $A$ and $C$ and the covariance matrices $Q$ and $R$, solving an algebraic Riccati equation [20, Chap. 23] yields a gain $L_{K}$ that "places" the eigenvalues of $A-L_{K} C$ in (15) in a specific location.

If we selected $L=L_{K}$ in (14), the positive definiteness of $Q$ and $R$ would imply that all the eigenvalues of $A-L_{K} C$ have negative real part. Our proposed selection of $L$ is described next, and will result in a suitable scaling of the error dynamics (15).

\section{B. "High"-gain enhancement}

From the estimation error $\tilde{x}_{i}:=\left[\begin{array}{llll}\tilde{v} & \tilde{a}_{i} & \tilde{o}_{a} & \tilde{J}_{i}\end{array}\right]^{T}$, we define the "high"-gain scaled error and the (inverse of the) diagonal matrix $\mathcal{L}$ as

$$
e:=\left[\begin{array}{c}
\tilde{v} \\
\frac{\tilde{a}_{i}}{\ell} \\
\frac{\tilde{\sigma}_{a}}{\ell} \\
\tilde{\tilde{\tilde{z}}} \\
\frac{\ell^{2}}{\ell^{2}}
\end{array}\right]=\left[\begin{array}{cccc}
1 & & & \\
& \frac{1}{\ell} & & \\
& & \frac{1}{\ell} & \\
& & & \frac{1}{\ell^{2}}
\end{array}\right] \tilde{x}_{i}=: \mathcal{L}^{-1} \tilde{x}_{i} .
$$

The scalar parameter $\ell$ in $\mathcal{L}$ is the tuning knob that allows the user to choose between responsiveness in the estimation and noise rejection. In other words, if $\ell$ is large, the convergence rate of the estimate is increased (for instance, if $L_{K}$ was tuned too conservatively); if $\ell$ is small, it improves the filtering action of the observer with respect to measurement noise. This role of $\ell$ is justified theoretically in the rest of this section, and is illustrated on experimental data in Section IV-B.

Remark 1: The name "high"-gain is motivated by the fact that a similar scaling in the inverse powers of a coefficient $\ell$ takes place in high-gain observers (for a full perspective on high-gain observers see, e.g., [18]). Historically, the parameter $\ell$ was chosen large (or "high") to dominate over the effect of uncertain or known nonlinearities.

Our proposed selection for the observer gain $L$ in (14) builds on the $L_{K}$ that stems from the Kalman formulation, and is parametrized by $\ell$ as

$$
L:=\left[\begin{array}{cccc}
1 & & \\
& \ell & \\
& & \\
& & \ell^{2}
\end{array}\right] L_{K}\left[\begin{array}{ll}
1 & \\
&
\end{array}\right] .
$$

We can then write the dynamics for the scaled error $e$ in (16). From (16) and (15), we get

$$
\dot{e}=\mathcal{L}^{-1}(A-L C) \mathcal{L} e .
$$

By the definition of $L$ in (17) and the peculiar structure of $A$ and $C$ in (11c), it follows immediately that $\mathcal{L}^{-1}(A-L C) \mathcal{L}=$ $\ell\left(A-L_{K} C\right)$, and hence

$$
\dot{e}=\ell\left(A-L_{K} C\right) e .
$$

Equation (18) demonstrates that the eigenvalues of the scaled error dynamics are exactly the eigenvalues "placed" with the Kalman approach in Section III-A for the matrix $A-L_{K} C$, scaled altogether in the complex plane by the factor $\ell$. Moreover, error $\tilde{x}_{i}$ undergoes the same change in its dynamics because it is related to $e$ by the similarity transformation in (16). Therefore, once the baseline gain $L_{K}$ is fixed, tuning $\ell$ allows for shifting in the left-hand half plane the whole set of the observer eigenvalues to the right (better noise rejection) or to the left (better estimate responsiveness) with respect to their original positions: this shifting is obtained by one parameter only. The effect of this trade-off will be evident in Figure 4 .

Remark 2: The intuition behind the error scaling in (16) is the customary one adopted in the high-gain literature. Since (a subpart of) state matrix $A$ corresponds to a triple integrator, subsequent states are in a simple integral relation. Then, scaling the error $\tilde{x}_{i}$ as in (16) implies that also the roots of the characteristic polynomial of its dynamics are scaled through $\ell . o_{a}$ is scaled conveniently in the same way as $a_{i}$ because they are algebraically related in (10f). 


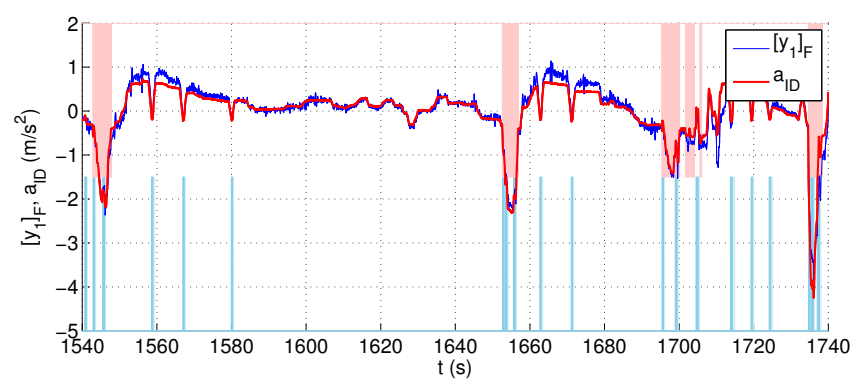

Figure 1. Signals $\left[y_{1}\right]_{\mathrm{F}}$ and $a_{\mathrm{ID}}$ for a typical portion of the validation half of the data. (The pink areas correspond to braking, the blue ones to gear shifts.)

\section{EXPERIMENTAL VALIDATION}

We validate our estimation approach on experimental data collected on a road test.

The test route was designed in a sufficiently rich manner and includes most of the possible road scenarios, such as urban arteries, extra urban roads, motorways, roundabouts, ramps, and intersections. The route starts from Centro Ricerche Fiat in Orbassano (Turin, Italy), drives through Piossasco, arrives at Pinerolo, and heads back to Orbassano on a partially different road. The total length is 53 kilometers of public roads and is driven by an ordinary driver in around 42 minutes.

The parameters needed for the proposed intentional jerk estimation amount to $M, \tau, c_{b}$ and $c_{r}$. The total mass of the vehicle is $M=1550 \mathrm{~kg}$, the trasmission coefficient $\tau$ is for the different gears $\tau_{1}=41, \tau_{2}=22, \tau_{3}=14, \tau_{4}=10, \tau_{5}=8$, and the remaining $c_{b}$ and $c_{r}$ are identified in Section IV-A. We note that we do not need to identify the parameters of the dissipative forces $F_{d}$ in (3) because we assumed that the typical driver is able to compensate for them, as we discussed after (5a).

The performance of our intentional jerk estimate is analyzed on experimental data in Sections IV-B-IV-D, but we emphasize that the filtering scheme can be utilized on line in the same form because neither the scheme nor the presented results entail any noncausal filtering.

\section{A. Identification of coefficients $c_{b}$ and $c_{r}$}

We address here the identification of $c_{r}$ and $c_{b}$, which we left in Section II-B: we consider first $c_{b}$ and then $c_{r}$.

As to $c_{b}$, when we substitute (2b) in (2a) with $F_{b}=c_{b} p_{b}$ from (8a), we obtain

$$
M\left(y_{1}-\bar{d}_{a}\right)-\tau T_{e}+c_{b} p_{b}=-C_{r} M g-\frac{1}{2} \rho S C_{x} v^{2}=:-\kappa_{1}-\kappa_{2} v^{2}
$$

where $y_{1}$ and $v$ are measured signals, $T_{e}$ and $p_{b}$ are known from the CAN bus of the vehicle, $M$ is a known parameter. Note that the gravitational term $g \alpha$ cancels out after the substitution, and in (19) we neglect $\bar{d}_{a}$ as compared to the other terms only for the purposes of this identification section. Then we are left with $c_{b}, \kappa_{1}, \kappa_{2}$ to identify.

Since we are interested in identifying the slow dynamics and the measurement $y_{1}$ is noisy, we filter (19). For a generic signal $w$ define its filtered version $[w]_{\mathrm{F}}:=\frac{1}{1+s /\left(2 \pi f_{\mathrm{LP}}\right)} w$ with $f_{\mathrm{LP}}=5 \mathrm{~Hz}$, so that (19) becomes

$$
M\left[y_{1}\right]_{\mathrm{F}}=\left[\tau T_{e}\right]_{\mathrm{F}}-c_{b}\left[p_{b}\right]_{\mathrm{F}}-\kappa_{1}-\kappa_{2}\left[v^{2}\right]_{\mathrm{F}} .
$$

Stacking all sampled values of the filtered signals, (20) results in a linear system in the unknowns $c_{b}, \kappa_{1}, \kappa_{2}$. We solve it in the least squares sense minimizing the mismatch between $M\left[y_{1}\right]_{\mathrm{F}}$ and the right-hand side in (20). As standard practice in identification, we divide the acquired data in two halves, and obtain the least square solution on the first half, to validate it on the second half. The identification leads to the values $c_{b}=189 \mathrm{~N} / \mathrm{bar}, \kappa_{1}=131 \mathrm{~N}, \kappa_{2}=0.283 \mathrm{~kg} / \mathrm{m}$. We present in Figure 1 the evolution of $\left[y_{1}\right]_{\mathrm{F}}$ compared to $a_{\mathrm{ID}}:=\frac{1}{M}\left(\left[\tau T_{e}\right]_{\mathrm{F}}-c_{b}\left[p_{b}\right]_{\mathrm{F}}-\kappa_{1}-\kappa_{2}\left[v^{2}\right]_{\mathrm{F}}\right)$ as an illustration of the validation of the identified coefficients. $\left[y_{1}\right]_{\mathrm{F}}$ and $a_{\mathrm{ID}}$ match well.

Although we divided the data in two halves for simplicity, much less data would have been needed to identify the coefficient $c_{b}$, which is the only relevant parameter for our estimation scheme. Indeed, in Figure 2, we represent how the identified coefficient $c_{b}$ varies as the amount of identification data (in minutes of acquisition) grows larger. 20 minutes (of data) correspond to taking the first half of the data as was done above, and the blue dashed lines correspond to $\pm 5 \%$ of the value of $c_{b}$ at 20 minutes. It is clear then from the figure that some minutes of data suffice for a suitable identification of $c_{b}$ resulting in a small enough value of the Root Mean Square Error RMSE $:=\sqrt{\frac{1}{N} \sum_{k=1}^{N}\left(\left[y_{1}\right]_{\mathrm{F}}\left(t_{i}\right)-a_{\mathrm{ID}}\left(t_{i}\right)\right)^{2}}$, where $t_{1}$ and $t_{N}$ refer respectively to the beginning and the end of the validation data.

As to $c_{r}$, we obtain it through the signals $T_{r}$ and $p_{r}$ that are plotted as blue points in Figure 3 . Their distribution justifies the selection of the linear dependence in (8a). By least squares fitting again, we find $c_{r}=3.25$.

As a final remark on this section, the identification part is proposed in order not to have to rely on sensitive data such as engine/brake maps and is completely indipendent of the estimation scheme for the intentional jerk. 


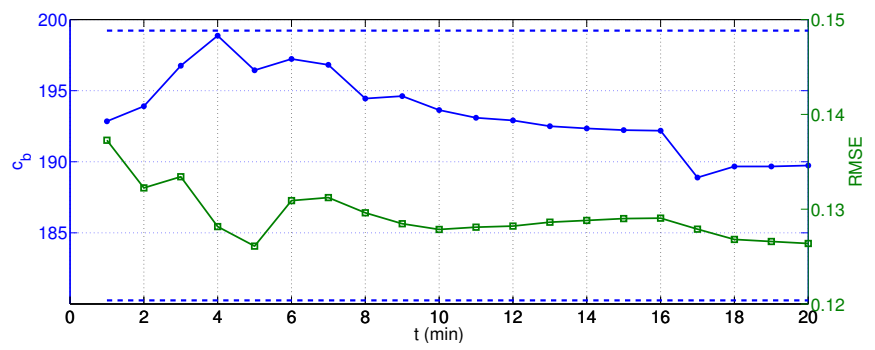

Figure 2. Evolution of the identified coefficient $c_{b}$ (blue) as a larger amount of identification data is taken, and corresponding evolution of the Root Mean Square Error defined in the text (green). The dashed blue lines correspond to $\pm 5 \%$ of the value of $c_{b}$ identified for 20 minutes.

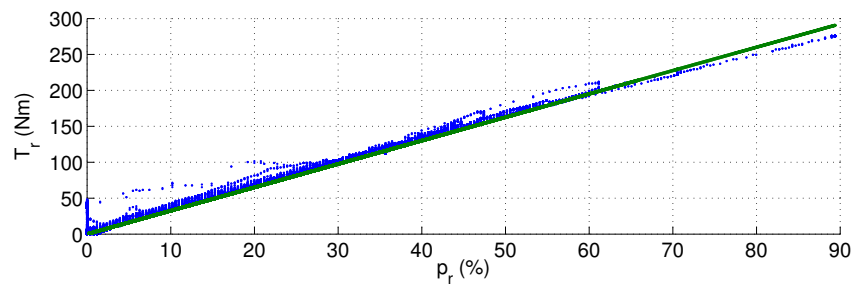

Figure 3. Signal $T_{r}$ plotted as a function of signal $p_{r}$ (blue). Linear approximant (green) providing parameter $c_{r}$.
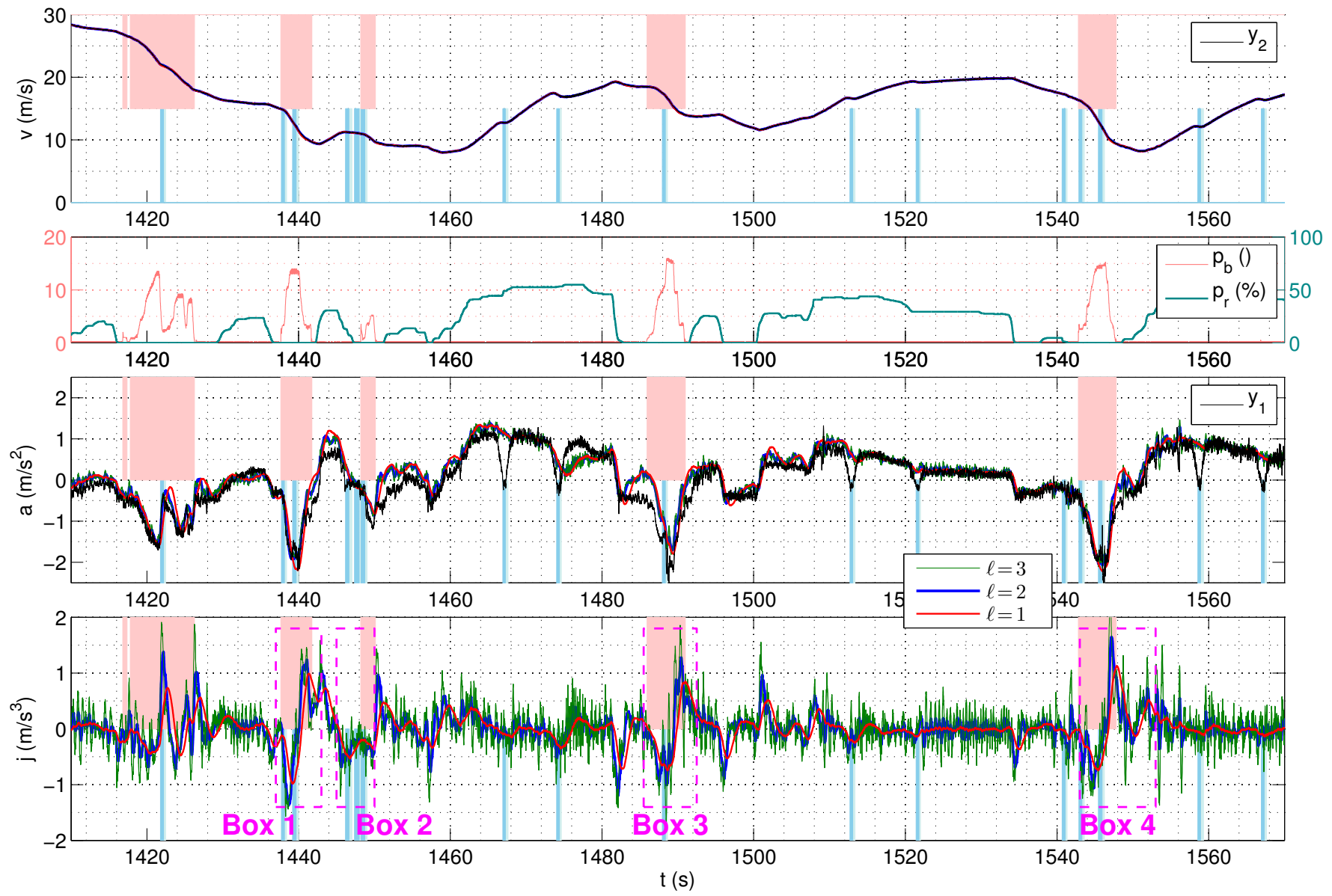

Figure 4. (First plot from the top) Velocity estimates $\hat{v}$ and measurement $y_{2}$. (Second) Cylinder brake pressure $p_{b}$ and gas pedal position $p_{r}$. (Third) Intentional acceleration estimates $\hat{a}_{i}$ and measurement $y_{1}$. (Fourth) Intentional jerk estimates $\hat{\bar{j}}_{i}$. As for the estimates, the colors green, blue, red correspond respectively to the values $3,2,1$ for the tuning knob $\ell$.

\section{B. Performance of our estimation scheme}

The covariance matrices in (13) were chosen as

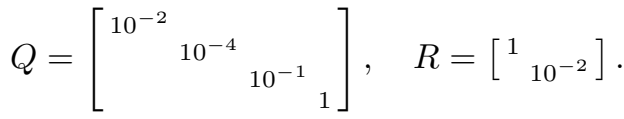



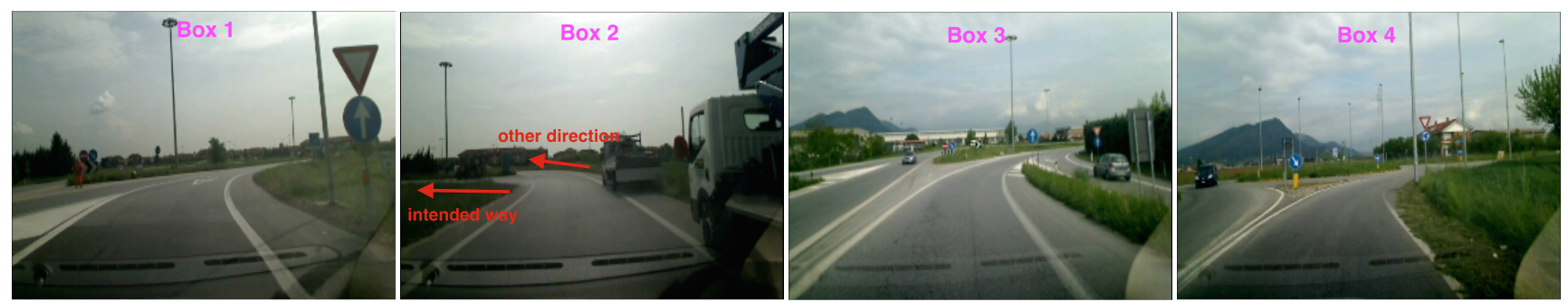

Figure 5. From left to right, shot $i$-th corresponds to the $i$-th magenta box in Figure 4 .

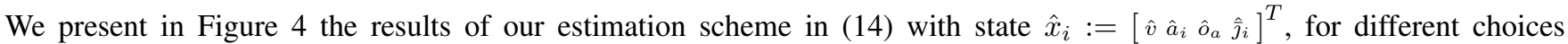
of $\ell$. All along the figure, pink areas represent times when the driver is braking, whereas blue areas represent times when a gear change occurs (darker blue when the clutch is disengaged from the engine, lighter blue when it is in the process of re-engagement).

At the top of the figure, the estimated velocity $\hat{v}$ is barely distinguishable from the measurement $y_{2}$ (black) as expected, thanks to the intrinsic reliability of $(10 \mathrm{~g})$, which also motivates the low value at the bottom corner of $R$ in (21). The portion of data has been chosen sufficiently varied, as the velocity evolution shows. In the middle-top trace, we present the signals $p_{b}$ (bluish-green) and $p_{r}$ (dark pink) that serve as input $u$ to (14) in addition to $T_{e}$ (see (10a)). The plotted signals $y_{2}, p_{b}, p_{r}$ should help interpret and motivate the intentional acceleration and jerk at the bottom.

In the middle-bottom trace, we present the estimated intentional acceleration $\hat{a}_{i} . \hat{a}_{i}$ differs noticeably from $y_{1}$ as the former aims at taking into account the driver intention, whereas the latter incorporates the vehicle dynamics. In particular, all the gear shifts (blue areas) that impact on $y_{1}$ are suitably rejected in $\hat{a}_{i}$ : see in particular the significant one at $1467 \mathrm{~s}$. This suggests indirectly that the time derivative of $y_{1}$, that is, the actual jerk, is in general not a good candidate to infer intentions. The evolution of $\hat{a}_{i}$ bears actually more resemblance to a suitably weighted sum of $p_{r}$ and $-p_{b}$. This sum is further compared with $\hat{a}_{i}$ in Section IV-C.

From (10c), the intentional jerk $\hat{\bar{j}}_{i}$ descends directly from $\hat{a}_{i}$, and based on the bottom part of Figure 4, we justify its ability to capture changes in (high-level) driving maneuvers. More specifically, we consider the most relevant bursts in the evolution of the estimated intentional jerk, in the magenta boxes. For each one of them, an image in Figure 5 captures the most relevant moment of the corresponding scenario. The first box corresponds to a situation where after driving along a straight exit ramp, the driver is on a curve that intersects a major road. Its first reaction is braking because s/he has to give way, but as soon as $\mathrm{s} / \mathrm{he}$ realizes that the major road is free, s/he changes its goal and switches to a strong acceleration to join the major road. Right after that, in the second magenta box, the driver sees that the major road is taking a sharp bend, and even if the velocity is increasing and the gas pedal is being pressed, $\hat{\bar{\jmath}}_{i}$ is anticipating this abrupt change, that is confirmed shortly afterwards by a brake intervention. The third box is also related to an intersection, but the driver is now on a single-lane main road, and sees to his/her right another car coming from a ramp in front of him/her. His/her reaction at $1485 \mathrm{~s}$ is to brake, but soon after $\mathrm{s} / \mathrm{he}$ realizes that the other driver is coming at (significantly) higher speed, s/he changes the goal back to accelerating: this maneuver is witnessed by the intentional jerk wobble from negative to positive. The fourth box refers to a roundabout. The driver arrives sufficiently close to the roundabout at $1543 \mathrm{~s}$ after a straight road segment. S/he then brakes in order to be able to stop and give way, as in the negative burst of $\hat{\bar{j}}_{i}$. When s/he realizes that no car is coming from his/her left in the roundabout, s/he accelerates again, as in the first positive burst of $\hat{\bar{\jmath}}_{i}$. The second positive burst corresponds to exiting the roundabout. As for the examples in Boxes 1-2 and 4, we notice that the influence of the road map is indirect since the road map first influences the driver, who then converts it into different evolutions of the pedal signals based on the path s/he wants to travel. Encoding the road information as a dynamical system and including it in the model-based observer is an interesting but nontrivial task and is left as future work.

Interestingly, one could associate the described jerk bursts to changes in the high level maneuver that the driver wants to pursue. For instance, for the third magenta box, the negative burst of $\hat{\bar{j}}_{i}$ can be related to a transition from a free-flow maneuver (the driver had no vehicle in front of him/her) to a follow-object one [7, §III.D.4)] when s/he notices the car to his right. Again, when the other car distances the driver due to the significantly higher velocity, the driver may revert to the free-flow maneuver [7, §III.D.4)], which corresponds to the positive burst in $\hat{\bar{\jmath}}_{i}$.

Although the portion of the data set was purposedly chosen to highlight a number of events to corroborate the validity of our estimation scheme, we note that the baseline value for the intentional jerk $\hat{\bar{J}}_{i}$ is zero, consistently with the minimization of the jerk squared [4], [5].

Finally, we note that the smoothing effect of the parameter $\ell$ is especially evident for the signal $\hat{\bar{j}}_{i}$ : a large value $(\ell=3)$ makes the estimate more reactive but also more prone to noise, whereas a small value $(\ell=1)$ achieves the converse, bearing a possibly significant delay for its use in intention inference. In order to have a causal estimate, this delay is the intrinsic price to pay for a smoother filtered signal, as discussed after (18). A good trade-off can be obtained with $\ell=2$. Note that we can design the diagonal elements of $Q$ and $R$ in (21) based on high level considerations, that is, how trustworthy the 

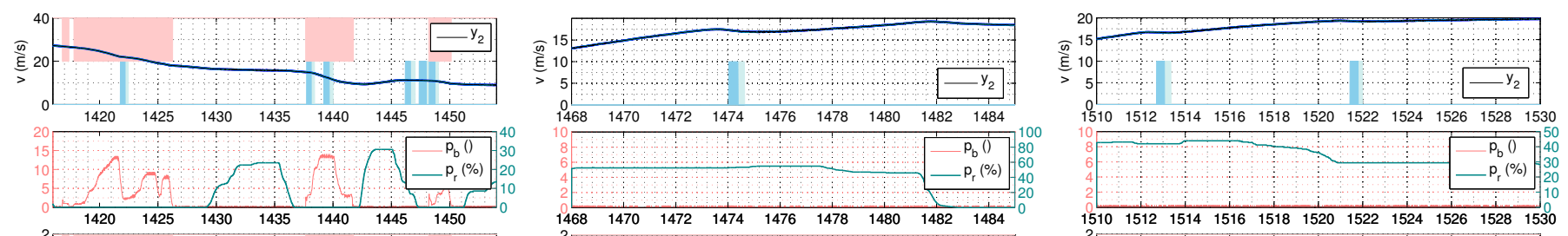

$$
\text { है }
$$
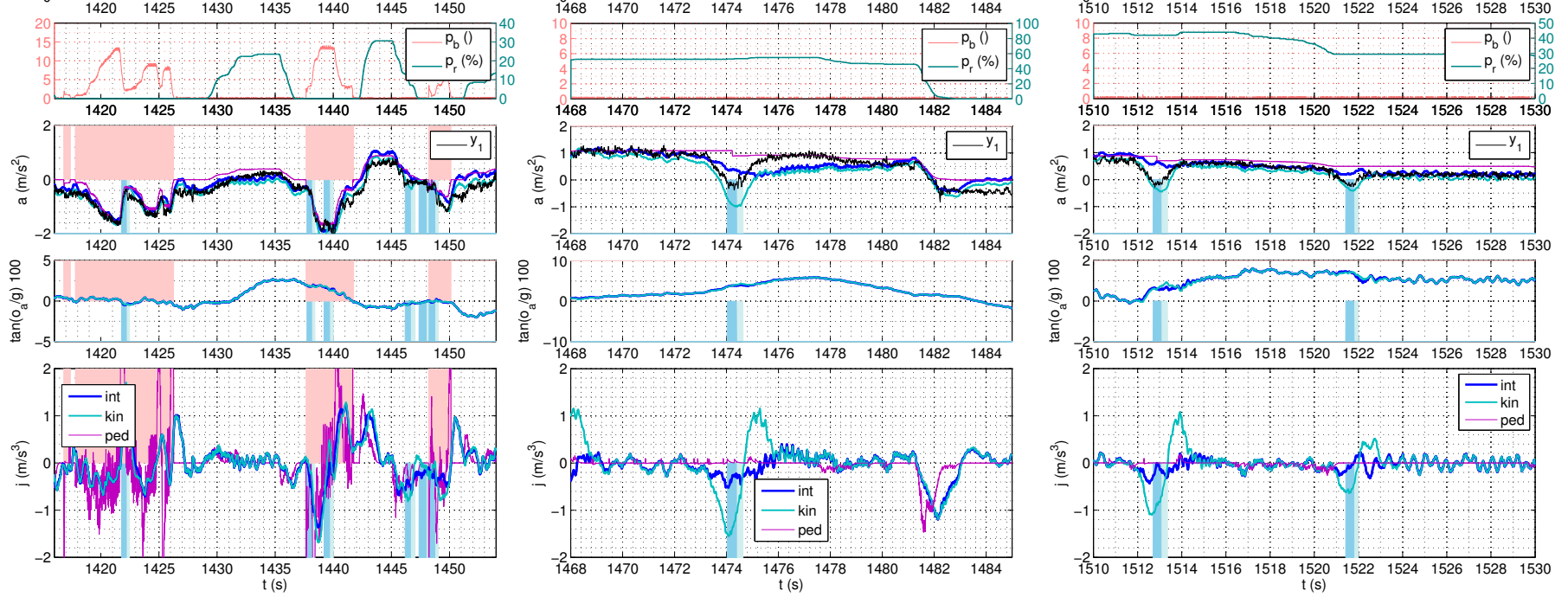

Figure 6. (First plots from the top) $\hat{v}$ for the intention and the kinematic model, and $y_{2}$. (Second) $p_{b}$ and $p_{r}$. (Third) $\hat{a}_{i}$ for the intentional model in blue, $\hat{a}$ for the kinematic model in turquoise, $a_{i}^{P}$ using the pedals in purple, and $y_{1}$ in black. (Fourth) $\hat{o}_{a}$ for the intention and the kinematic model, in blue and turquoise. (Fifth) $\hat{\bar{\jmath}}_{i}$ for the intentional model in blue, $\hat{\bar{j}}$ for the kinematic model in turquoise, $\bar{\jmath}_{i}^{P}$ using the pedals in purple.

(Left column plots) Sub-segment of Figure 4. (Center) Short uphill segment. (Right) Straight segment.

corresponding equation is as discussed after (10e), and then refine this choice by $\ell$. In this sense, $\ell$ overcomes partially the commonly perceived limitation that matrices $Q$ and $R$ in (13) are unknown in most cases.

\section{Comparison with other solutions}

We compare now the performance of our estimation scheme with two other possible solutions for estimating the intentional jerk. These solutions are shown to agree with our scheme, although having some limitations.

The first one is a kinematic model for the vehicle with state equations

$$
\dot{v}=a, \dot{a}=\bar{\jmath}, \dot{o}_{a}=0, \dot{\jmath}=0
$$

and output equations

$$
y_{1}=a+o_{a}, y_{2}=v
$$

Model (22) can be used straightforwardly in place of (10) in the enhanced Kalman formulation of Section III (for more details, see [19]), and was implemented in [7, Eq. (27)] to discriminate which one of the maneuvers hypothesized by the co-driver was the most likely one to be pursued by the driver him/herself. However, when the objective is to estimate the intentional jerk (see beginning of Section II-B), the mere use of $y_{1}$ (without the term $u$ in (10a)) is in principle misleading because $y_{1}$ reflects the actuated torque $T_{e}$ (and not $T_{r}$ ), and the whole vehicle dynamics.

The second solution disregards the compensation of the dissipation effects by the driver, and equates directly the pedal actions with the intentional accelerations (cf. (5)), that is,

$$
M \dot{v}_{i, 0}^{P}=\tau c_{r} p_{r}, M \dot{v}_{i, 1}^{P}=-c_{b} p_{p}
$$

where superscript ${ }^{P}$ denotes that this approach is based only on pedals. With (4), and parallel to (6), we write

$$
a_{i}^{P}:=(1-\sigma) \dot{v}_{i, 0}^{P}+\sigma \dot{v}_{i, 1}^{P},
$$

and using the jerk definition $\bar{\jmath}_{i}^{P}=\dot{a}_{i}^{P}$, we have from (23a) and (23b) that

$$
\bar{\jmath}_{i}^{P}=(1-\sigma) \frac{\tau}{M} c_{r} \dot{p}_{r}-\sigma \frac{1}{M} c_{b} \dot{p}_{b},
$$

almost everywhere, as the transmission coefficient $\tau$ is a piecewise constant signal. Even not differentiating $\tau$, the presence of noise in $p_{r}$ and $p_{b}$ requires an approximate time differentiation as in

$$
\bar{\jmath}_{i}^{P}=(1-\sigma) \frac{\tau}{M} c_{r} p_{r}^{\circ}-\sigma \frac{1}{M} c_{b} p_{b}^{\circ}
$$

where $p_{\{r, b\}}^{\circ}=\frac{s}{1+s /\left(2 \pi f_{\mathrm{D}}\right)} p_{\{r, b\}}$ and $f_{\mathrm{D}}$ was chosen as $2 \mathrm{~Hz}$. 
We choose three illustrative cases in Figures 6 to show that the two solutions (22)-(23) agree with our estimation, although they have some limitations. Specifically, we take for our solution the previous optimal value of $\ell=2$, and to have a fair comparison we tune with the same parameter the enhanced Kalman version based on the kinematic model (22). The pedal solution is already determined completely by (23b) and (23c).

Figure 6 (left column) shows agreement between our solution and the kinematic model (22) (more precisely, the enhanced Kalman scheme based on it), and underscores the main limitation of approach (23), which is prone to errors induced by numerical differentiation. Note that the spikes are due entirely to $p_{r}$ and, more evidently, to $p_{b}$, and are still present in spite of (heavy) filtering of differentiation $\left(f_{\mathrm{D}}=2 \mathrm{~Hz}\right.$ above). On the other hand, our solution has a similar reactiveness to change, without spikes.

Figure 6 (center column) shows the driver reaction when driving on a short uphill segment. The presence of an increasing road slope is well witnessed by the variable $\hat{o}_{a}$ of both the enhanced Kalman formulations, and $\tan \left(\frac{o_{a}}{g}\right) \cdot 100$ is depicted in the fourth plot from top of Figure 6 (center column). The driver is aware of the slope when determining his/her action, but since the segment is short, s/he decides not to intervene on the pedals even though this would lead to a decrease in his/her cruise speed, and this causes a small negative jerk. In this sense, our solution conforms better with the situation with respect to the differentiation of the pedals. The kinetic model suffers from the gear shift induced by the increased slope.

Figure 6 (right column) is associated to a slightly inclined straight segment, and shows the main limitation of the kinematic model (22) in estimating the driver intentions. Indeed, (22) includes altogether all the effects related to the longitudinal dynamics, most notably the deceleration/acceleration induced by the powertrain during a gear shift. This gives rise to the jerk bursts at 1513 and $1522 \mathrm{~s}$, that may be mistakenly associated to a change of intentions of the driver. If this kinematic estimation scheme is approximately taken as equivalent to a jerk measurement, its limits in estimating the driver intentions suggest then that there is no simple way such as a (possibly costly) sensor that can be used as ground truth to validate the quality of our estimate, which must then be assessed by considering the context the driver is in, as we did in Sections IV-B-IV-C.

\section{Comparison with different drivers}

We finally use our estimation scheme to obtain the intentional jerk for six different drivers. The driving style clearly affects the jerk evolution because it affects the pedal positions $p_{r}$ and $p_{b}$ in the first place, which are inputs for our estimation scheme. The results presented here show that we can capture the driver intentions through the proposed jerk estimate and it is emphasized that different driving styles lead to distinct jerk estimates, as shown below.

We consider for the different drivers the same road portion of Figure 4. In order to compare the evolutions effectively, we parametrize the data with respect to the curvilinear coordinate $s$, instead of time $t$. The results are shown in Figure 7. The first three drivers are on the left, and the second three are on the right. The traces associated to the same driver are in the same color, as specified in the legends, and we group in a same plot the evolutions of some variables relative to the first three and the second three drivers (the velocity $v$, the gas pedal position $p_{r}$, the cylinder brake pressure $p_{b}$, the estimated intentional acceleration $\hat{a}_{i}$ and the estimated slope $\tan \left(\frac{\hat{o}_{a}}{g}\right) \cdot 100$ ), whereas we devote a single plot to the evolution of the estimated jerk $\hat{\bar{j}}_{i}$ for each driver. All evolutions in dark blue (corresponding to the sixth driver) coincide with the ones of Figure 4 for $\ell=2$, after reparametrizing them with the curvilinear coordinate $s$.

As in the previous sections, we propose the measured variables $v, p_{r}$ and $p_{b}$ for illustration at the top of Figure 7. In the central part of Figure 7 we show the estimated intentional accelerations $\hat{a}_{i}$ for each driver triplet, which present similar evolutions induced by the road profile. The estimated slope of the road as a function of the curvilinear coordinate $s$ coincides significantly for the six drivers, showing the reliability of our estimation scheme. The same enhanced Kalman filter was adopted for all the six drivers, with $Q$ and $R$ as in (21) and $\ell=2$, which was deemed the optimal value in Section IV-B. With these parameters, the evolutions of the estimated jerk are in the bottom part of Figure 7, where we surround with magenta boxes the same road sub-portions highlighted in Figure 4 and 5. A total length of $2.3 \mathrm{~km}$ is analyzed.

We can now comment in detail the jerk evolutions. The descriptions of the scenarios in the boxes were provided in Section IV-B. Based on those, we can see that in the first box all the drivers present a very similar evolution of the estimated jerk that is associated to first decelerating (and possibly triggering a full stop maneuver as for driver 5) to give way at the exit ramp, and then accelerating again to adapt to the higher speed of the main road. The second driver is the one anticipating the most the deceleration phase. In the second box we also witness for all the drivers a similar negative burst in the jerk due to the sharp bend. The scenario in the third box is quite peculiar to the sixth driver, because of the maneuvre of the vehicle in front of him/her (refer to Section IV-B), so no strong similiarities among the estimated jerks of the drivers are expected and found. Finally, in the fourth box (corresponding to a roundabout) the sixth and third drivers present a very similar pattern (a negative jerk burst for the stop and then two positive ones for the entrance and exit from the roundabout). Such a pattern is to be seen also for the second and the fifth driver by noticing that, again, the second driver tends to anticipate significantly the deceleration phase as in the first box. If we look more generally at the whole evolution, the first driver (and partially the fourth) uses the pedals quite aggressively as we see from the light blue trace in the second and third left plots from the top. This produces, in turn, a similarly nervous intentional acceleration and jerk evolution, where many bursts consistently appear. On the other hand, the fifth driver uses the pedals much more smoothly, keeping them constant on large intervals of time or 

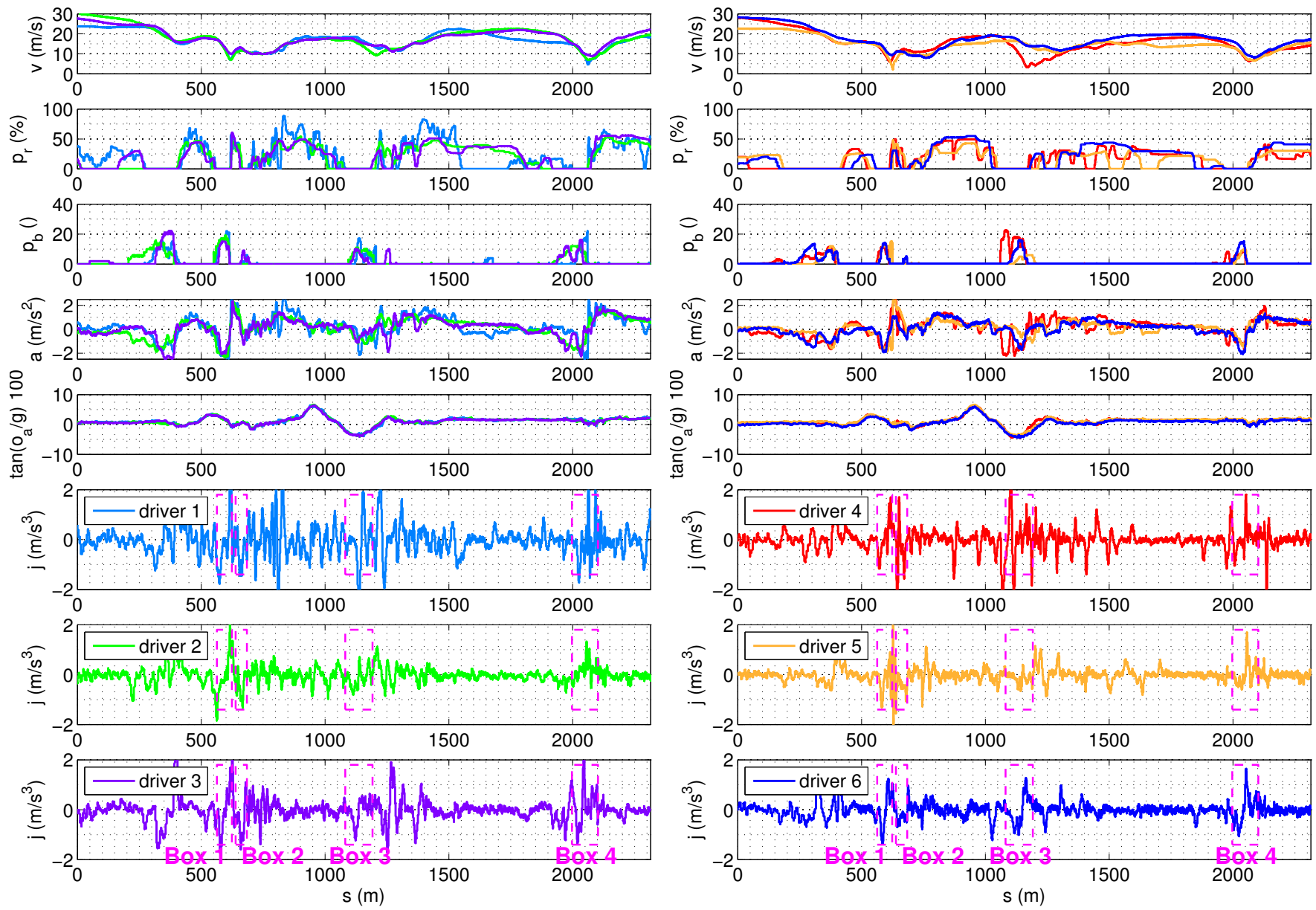

Figure 7. Evolution for different drivers of the measured variables $\left(v, p_{r}, p_{b}\right)$ and the estimated variables (intentional acceleration $\hat{a}_{i}$, slope $\hat{o}_{a}$, intentional jerk $\hat{\bar{j}}_{i}$ ) with respect to the curvilinear coordinate $s$. A separate plot is allocated to the $\hat{\bar{j}}_{i}$ of each of the six drivers in the part below, and the traces in the part above associated to the same driver are in the same color (as specified in the legends).

varying them in an approximately linear fashion. This consistently results in a much more limited jerk evolution. The third and sixth drivers present intermediate jerk evolutions between these two extreme cases.

Summarizing, we emphasize that the driving style has no role in the design of our estimation scheme, which is the core interest of the present work, and that different driving styles are successfully detected by our estimator, which can then be used to infer driver intentions.

\section{CONCLUSIONS AND FUTURE WORK}

This work addressed the problem of estimating the longitudinal jerk of a vehicle as it is acted by the typical human driver. We provided first an analytical justification for the intention-oriented model of the longitudinal dynamics, and then showed the effectiveness of the proposed enhanced Kalman scheme on experimental data, comparing it with alternative approaches and for different drivers.

Future work will consider placing this scheme directly in the advanced driver assistance system that uses the jerk estimate to infer the driver intention. The scheme is indeed ready for an on-line implementation because it does not use any noncausal information. It could also be generalized to estimate the intentional lateral jerk. Other possible directions for future work may include: substituting the enhanced Kalman approach with a different filtering paradigm (like particle filters), using the information from the road map directly in the filter, and employing the jerk estimate to carry out naturalistic driving studies and to find the average distribution of the longitudinal jerk for different people and roads, or to classify different driving styles as in [26].

\section{ACKNOWLEDGMENT}

The research leading to these results has received funding from the European Commission Seventh Framework Programme (FP/2007-2013) under the projects InteractIVe, grant agreement number 246587, and AdaptIVe, grant agreement number 610428. Responsibility for the information and views set out in this article lies entirely with the authors. The authors would like to 
thank all partners within InteractIVe and AdaptIVe for their cooperation and valuable contribution. The work has also been supported by the University of Trento, grant OptHySYS. The authors would like to thank Andrea Saroldi and Centro Ricerche Fiat for their contribution in collecting the experimental data, and Giulio Panzani and Marco Galvani for useful discussions.

\section{REFERENCES}

[1] T. Flash and N. Hogan, "The coordination of arm movements: an experimentally confirmed mathematical model," The Journal of Neuroscience, vol. 5, no. 7, pp. 1688-1703, 1985.

[2] P. Viviani and T. Flash, "Minimum-jerk, two-thirds power law, and isochrony: converging approaches to movement planning." Journal of Experimental Psychology: Human Perception and Performance, vol. 21, no. 1, p. 32, 1995.

[3] C. M. Harris, "Biomimetics of human movement: functional or aesthetic?" Bioinspiration \& biomimetics, vol. 4, no. 3 , p. $033001,2009$.

[4] T. Hiraoka, T. Kunimatsu, O. Nishihara, and H. Kumamoto, "Modeling of driver following behavior based on minimum-jerk theory," in Proc. 12th World Congress ITS, 2005

[5] M. Yamakado and M. Abe, "An experimentally confirmed driver longitudinal acceleration control model combined with vehicle lateral motion," Vehicle System Dynamics, vol. 46, no. S1, pp. 129-149, 2008.

[6] M. Yamakado, J. Takahashi, S. Saito, A. Yokoyama, and M. Abe, "Improvement in vehicle agility and stability by G-Vectoring control," Vehicle System Dynamics, vol. 48, no. sup1, pp. 231-254, 2010.

[7] M. Da Lio, F. Biral, E. Bertolazzi, M. Galvani, P. Bosetti, D. Windridge, A. Saroldi, and F. Tango, "Artificial co-drivers as a universal enabling technology for future intelligent vehicles and transportation systems," IEEE Trans. Intell. Transp. Syst., vol. 16, no. 1, pp. 244-263, 2015.

[8] "interactIVe: accident avoidance by intervention for Intelligent Vehicles," Online resource, 2010-2013, available at http://www.interactive-ip.eu/ [Accessed September 26, 2016].

[9] Y. Demiris, "Prediction of intent in robotics and multi-agent systems," Cognitive processing, vol. 8, no. 3, pp. $151-158,2007$.

[10] M. Yamakado and M. Abe, "Examination of voluntary driving operational timing by using information obtained with the developed jerk sensor," in FISITA 2006, 2006.

[11] J.-J. Martinez and C. Canudas-de Wit, "A safe longitudinal control for adaptive cruise control and stop-and-go scenarios," IEEE Trans. Control Syst. Technol., vol. 15, no. 2, pp. 246-258, 2007.

[12] J. J. Rangel-Magdaleno, R. J. Romero-Troncoso, R. A. Osornio-Rios, and E. Cabal-Yepez, "Novel oversampling technique for improving signal-toquantization noise ratio on accelerometer-based smart jerk sensors in CNC applications," Sensors, vol. 9, no. 5, pp. 3767-3789, 2009.

[13] L. Morales-Velazquez, R. de Jesus Romero-Troncoso, R. A. Osornio-Rios, and E. Cabal-Yepez, "Sensorless jerk monitoring using an adaptive antisymmetric high-order FIR filter," Mechanical Systems and Signal Processing, vol. 23, no. 7, pp. 2383 - $2394,2009$.

[14] A. M. Dabroom and H. K. Khalil, "Discrete-time implementation of high-gain observers for numerical differentiation," International Journal of Control, vol. 72 , no. 17 , pp. 1523-1537, 1999.

[15] Y. Chitour, "Time-varying high-gain observers for numerical differentiation," IEEE Trans. Autom. Control, vol. 47, no. 9, pp. 1565-1569, 2002.

[16] S.-i. Nakazawa, T. Ishihara, and H. Inooka, "Real-time algorithms for estimating jerk signals from noisy acceleration data," International Journal of Applied Electromagnetics and Mechanics, vol. 18, no. 1, pp. 149-163, 2003.

[17] C. Guo, C. Sentouh, B. Soualmi, J.-B. Haué, and J.-C. Popieul, "Adaptive vehicle longitudinal trajectory prediction for automated highway driving," in 2016 IEEE Intelligent Vehicles Symposium, 2016, pp. 1279-1284.

[18] H. K. Khalil and L. Praly, "High-gain observers in nonlinear feedback control," International Journal of Robust and Nonlinear Control, vol. 24, no. 6, pp. 993-1015, 2014.

[19] A. Bisoffi, F. Biral, M. Da Lio, and L. Zaccarian, "Longitudinal jerk estimation for identification of driver intention," in 18th IEEE International Conference on Intelligent Transportation Systems, 2015, pp. 1855-1861.

[20] J. P. Hespanha, Linear Systems Theory. Princeton, New Jersey: Princeton Press, 2009.

[21] E. Todorov and M. I. Jordan, "A minimal intervention principle for coordinated movement," Advances in neural information processing systems, pp. 27-34, 2003.

[22] P. Lingman and B. Schmidtbauer, "Road slope and vehicle mass estimation using Kalman filtering," Vehicle System Dynamics, vol. 37, no. sup1, pp. $12-23,2002$.

[23] Y. Sebsadji, S. Glaser, S. Mammar, and J. Dakhlallah, "Road slope and vehicle dynamics estimation,” in American Control Conference, 2008, 2008, pp. 4603-4608.

[24] M. Klomp, Y. Gao, and F. Bruzelius, "Longitudinal velocity and road slope estimation in hybrid electric vehicles employing early detection of excessive wheel slip," Vehicle System Dynamics, vol. 52, no. sup1, pp. 172-188, 2014.

[25] M. Corno, P. Spagnol, and S. M. Savaresi, "Road slope estimation in bicycles without torque measurements," Proc. 19th IFAC Word Congress, pp. 6295-6300, 2014

[26] Y. L. Murphey, R. Milton, and L. Kiliaris, "Driver's style classification using jerk analysis," in IEEE Workshop on Computational Intelligence in Vehicles and Vehicular Systems. IEEE, 2009, pp. 23-28.

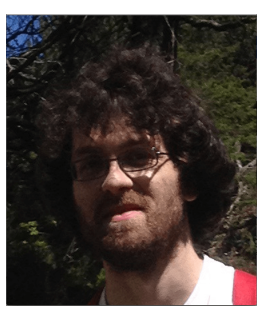

Andrea Bisoffi received his M.Sc. with honors in Automatic Control Engineering from Politecnico di Milano, Italy, in 2013 and his $\mathrm{Ph}$. D. in Mechatronics from the University of Trento, Italy, in 2017. In 2015-2016 he was a visiting scholar in the Control Group at the University of Cambridge, UK. His current research interests include hybrid and nonlinear control systems, with applications to mechanical and automotive systems. 


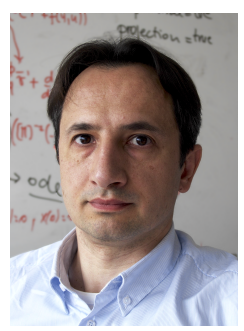

Francesco Biral was born in Italy, on February $2^{\text {nd }}$, 1972. In 1997 he received the Master Degree in Mechanical Engineering at the University of Padova, Italy, and the Ph.D. in Mechanism and Machine Theory from University of Brescia, Italy, in 2000. He is currently Associate Professor at the Department of Industrial Engineering at University of Trento. His research interests include symbolic and numerical multibody dynamics and optimisation, constrained optimal control, mainly in the field of vehicle dynamics with special focus on Intelligent Transportation Systems.

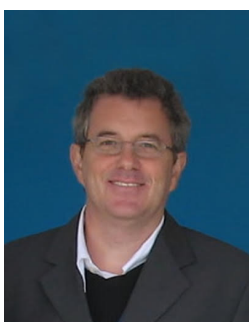

Mauro Da Lio received the Laurea degree in mechanical engineering from the University of Padova, Italy, in 1986. He is Full professor of mechanical systems with the University of Trento, Italy. His earlier research activity was on modeling, simulation and optimal control of mechanical multibody systems, in particular vehicle and spacecraft dynamics. More recently his focus shifted to the modeling of human sensory-motor control, in particular drivers and motor impaired people. Prior of academic carrier he worked for an offshore oil research company in underwater robotics (an EUREKA project). He was/is involved in several EU framework programme 6 and 7 projects (PReVENT, SAFERIDER, interactIVe, VERITAS, adaptIVe, and NoTremor) and he is coordinator of Horizon2020 Dream4Cars.

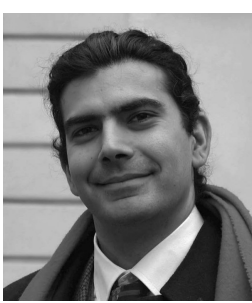

Luca Zaccarian has been Assistant and then Associate Professor at the University of Roma Tor Vergata (Italy) since 2000. Then in 2011 he became Directeur de Recherche at the LAAS-CNRS, Toulouse (France) and since 2013 he also holds a part-time associate professor position at the University of Trento, Italy. Luca Zaccarian's main research interests include analysis and design of nonlinear and hybrid control systems, modeling and control of mechatronic systems. He was a recipient of the 2001 O. Hugo Schuck Best Paper Award given by the American Automatic Control Council and he is a fellow of the IEEE. 